\title{
Improved Cable Insulation for Superconducting Magnets*
}

\author{
M. Anerella, A.K.Ghosh, E. Kelly, J. Schmalzle, E. Willen \\ Brookhaven National Laboratory \\ Upton, New York 11973 \\ J. Fraivillig, J. Ochsner, D.J. Parish \\ E. I. DuPont de Nemours \& Co. \\ Wilmington, Delaware
}

\section{INTRODUCTION}

Several years ago, Brookhaven joined with DuPont in a cooperative effort to develop improved cable insulation for SSC superconducting dipole magnets. The effort was supported by the SSC Central Design Group and later the SSC Laboratory. It was undertaken because turn-to-turn and midplane shorts were routinely being experienced during the assembly of magnets with coils made of the existing Kapton" /Fiberglass (K/FG) system of Kapton film overwrapped with epoxy-impregnated fiberglass tape. Dissection of failed magnets showed that insulation disruption and punch-through was occurring near the inner edges of turns close to the magnet midplane. Coil pressures of greater than $17 \mathrm{kpsi}$ were sufficient to disrupt the insulation at local high spots where wires in neighboring turns crossed one another and where the cable had been strongly compacted in the keystoning operation during cable manufacture.

In the joint development program, numerous combinations of polyimide films manufactured by DuPont with varying configurations and properties (including thickness) were subjected to tests at Brookhaven. Early tests were bench trials using wrapped cable samples. The most promising candidates were used in coils and many of these assembled and tested as magnets in both the SSC and RHIC magnet programs currently underway. The Kapton $\mathrm{CI}(\mathrm{CI})$ system that has been adopted represents a suitable compromise of numerous competing factors. It exhibits improved performance in the critical parameter of compressive punch-through resistance as well as other advantages over the K/FG system:

- Superior manufacturability

- Relaxation of product storage requirements

- Reduced coil curing time

- Improved accommodation of component size variation

- Reduced mold cleanup after cure

- Improved coil repair/rework capability

- Increased radiation resistance

- Retention of ductility at cryogenic temperature

- Improved conductor placement uniformity

Figure 1 shows the K/FG system in common use and the new Cl system. A technical description of the construction process for SSC magnets is given in Ref. 1 .

\footnotetext{
* Work performed under Contrace No. DE-ACO2-76ClkXX16 with the U.S. Department of Energy.

* Kapten is a registered trademark of E.I. DuPont de Nernours \& Co.
}

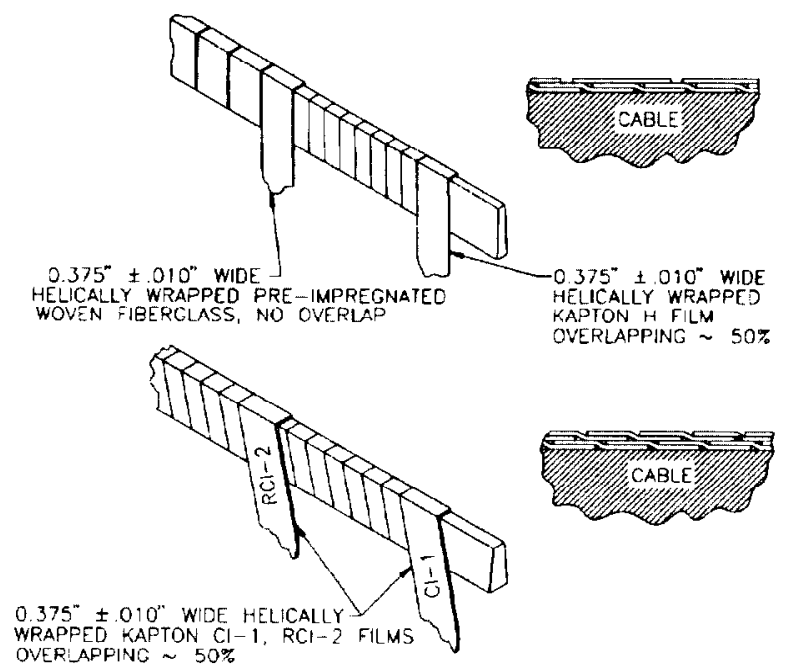

Figure 1. Kapton/Fiberglass insulation (top) and Kapton CI insulation (bottom) as applied to superconducting cable.

\section{DESCRIPTION}

Kapton $\mathrm{CI}$ is a $100 \%$ polyimide cable insulation system. The inner wrap, $120 \mathrm{Cl}-1$, is a $0.001^{\prime \prime}$ thickness amorphous Kapton film with $0.0002^{\prime \prime}$ thickness polyimide adhesive on the outside surface. The outer wrap, $135 \mathrm{RCI}-2$, is a $0.00095^{\prime \prime}$ thickness mineral-filled amorphous Kapton film with a total thickness of 0.0004 " polyimide adhesive, half on each surface. The adhesive is composed of aromatic diamines plus aromatic and co-aromatic dianhydrides. This all-aromatic product ensures ductility at cryogenic temperature and has superior resistance to ionizing radiation as compared to a system that uses epoxy. Upon reaching a temperature of $217 \mathrm{C}$, the adhesive bonds in several seconds to form a strong bond (peel strength $\sim 0.2 \mathrm{lb}$. per inch of width, about half that of $\mathrm{K} / \mathrm{FG}$ ), although some bonding occurs at lower temperatures. It flows out from areas of high compression to fill adjacent voids in the coil structure, thus forming a structure that spreads the compressive coil load over a wider area. It does not, however, lose viscosity nor flow into areas without compressive force. Photomicrographs of coil sections show that the Kapton films and adhesive of this system have, after a coil is molded, merged into a uniform matrix of polyimide material that partially fills the voids and interstitial spaces normally seen in cross sections of coil structure. The adhesive shows no tencency to bond to the wires of the cable, an undesirable 
characteristic that could lead to premature magnet quenching if it occurred. Coils made with $\mathrm{Cl}$ have a per turn thickness $\sim 0.00075^{\prime \prime}$ greater than those made with K/FG.

\section{TESTS \& CHARACTERISTICS}

Compressive punch-through tests were performed with the apparatus shown in Fig. 2. Results of numerous tests can be summarized as follows: CI cables fail in the range of 100 to $150 \mathrm{kpsi}$, depending on molding history; K/FG cables fail in the range 30 to $40 \mathrm{kpsi}$. These bench tests are confirmed by the experience with coils and magnets built with $\mathrm{Cl}$ : no compressive failures have been experienced in over 20 short (1 to $2 \mathrm{~m}$ ) magnets of various types nor in 4 long ( $10 \mathrm{~m}$ or more) SSC and RHIC dipole magnets. Particularly compelling were the results from coil sections that were compressed with $10 \mathrm{kpsi}$ pressure and that then had turn-toturn voltage applied to each pair of neighboring turns: $\mathrm{Cl}$ coils were able to withstand over $5 \mathrm{kV}$ without failure whereas K/FG coils routinely failed by arcing before $2 \mathrm{kV}$ was achieved and before full pressure was applied.

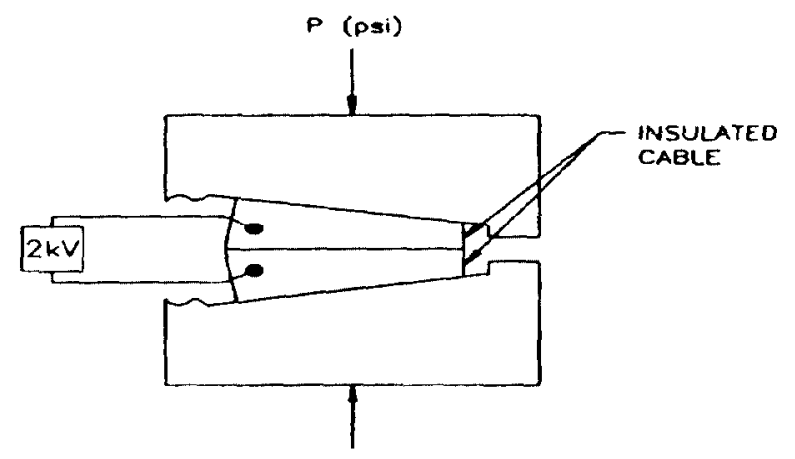

Figure 2. Apparatus for conducting compressive punch-through tests.

A desirable characteristic in the manufacture of coils is the capability to accommodate component (cable, wedge, insulation) thickness variation in such a way that final coil azimuthal size variation is small. This helps to ensure that magnets can be assembled within the required prestress window and that the magnet's field is uniform. Fig. 3 shows the range that was achieved for RHIC arc quadrupole coils (16 turns). Here, it was possible to achieve final coil sizes

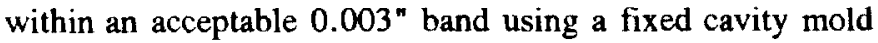
despite (intentional) cable thickness variation of $0.00125^{\text {" }}$ by simply varying the coil molding pressure over the range 10.5 to $12.9 \mathrm{kpsi}$. Alternatively, it was found that for constant cable thickness, the molded coil size could be varied over a range of 0.012 " with a molding pressure variation of $6 \mathrm{kpsi}$ (Fig. 4). Coil modulus varied $\sim 10 \%$ for this range of molding pressure. Thus the proper size for CI coils is easily achieved by proportionally adjusting the molding cavity size opposite to the sum of the variations of component sizes, as determined through incoming inspection data. These results cannot be achieved with K/FG coils. For such coils, coil-to- coil size uniformity can only be achieved for a more limited range of component thickness variation and by using a "double step" process in which molding cavity size is adjusted based on a coil size measurement made during the molding process. A related characteristic is the uniformity of coil azimuthal size along the length of a coil. While strongly tooling dependent, it is found that $\mathrm{CI}$ coils are equal to or improved in this important feature with respect to $\mathrm{K} / \mathrm{FG}$ coils.

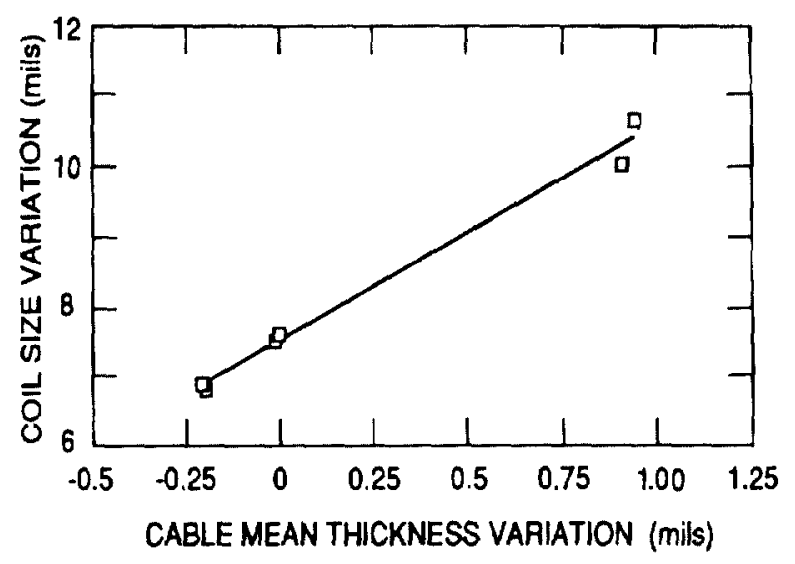

Figure 3. Kapton Cl molded coil size variation with variation in cable thickness. The allowable cable thickness variation is \pm 0.25 mils.

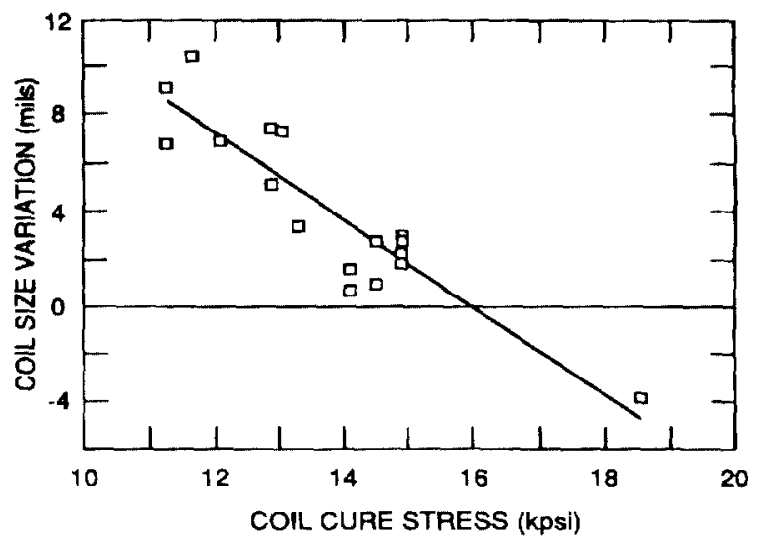

Figure 4. Kapton CI coil azimuthal size variation resulting from variation in the curing stress.

The compressive modulus of $\mathrm{CI}$ coils is found to be on the order of $1200 \mathrm{kpsi}$ for $8 \mathrm{~cm}$ RHIC dipole coils. This is about the same as that of $\mathrm{K} / \mathrm{FG}$ coils and it is quite suitable for magnet assembly. Fig. 5 shows the stress vs. strain relation for an SSC $50 \mathrm{~mm}$ inner coil. The higher resistance of $\mathrm{CI}$ coils to compressive insulation failure gives a safe window for magnet assembly stress of at least $10 \mathrm{kpsi}$, more than twice that which is safe for $\mathrm{K} / \mathrm{FG}$ coils. This is highly desirable in a production setting where component size variations within legitimate tolerances must be expected. An 
experiment to test coils built with all-polyimide film but using epoxy (3M-2290), which might give improved punch-through resistance without the need for molding at as high a temperature, gave a relatively lower modulus of $750 \mathrm{kpsi}$ and lower punch-through resistance values as compared to $\mathrm{CI}$ coils.

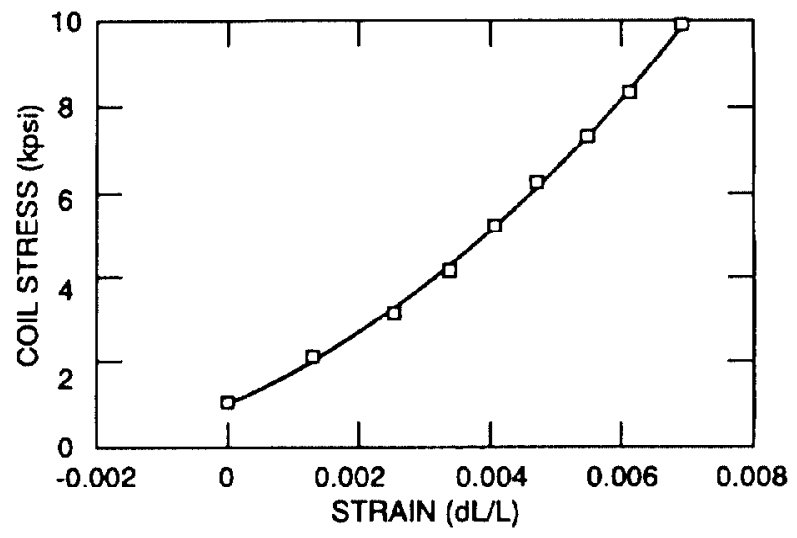

Figure 5. Stress vs. strain for a Kapton CI SSC 50 $\mathrm{mm}$ inner coil.

An early concern about coils made with all-polyimide cable insulation was that during a magnet quench, helium might be trapped inside the cable insulation and rupture the insulation as the pressure of the gas increased. Tests were performed on molded coil sections under conditions simulating those in a magnet to measure the impedance to the flow of helium from within the coil. This was done by fitting coil sections with buffer volumes at each end and measuring the flow of helium under various pressures out from the sections while the sections were compressed as they would be in a coil. It was found that the all-polyimide sections leaked helium at approximately $1 / 7$ the rate as the very porous $\mathrm{K} / \mathrm{FG}$ sections $^{[2]}$, but still sufficient to alleviate concern. In addition, a magnet that had been built with spot heaters to initiate quenching was extensively tested and then dissected to look for insulation damage; none was found. Thus, there is no concern that trapped helium can cause insulation damage during magnet quenching.

Another concem with early all-polyimide coils was that the increased amount of plastic material in these coils would lead to increased loss of coil prestress during cool down to operating temperature. Indeed, it was found that magnets made with early versions of the new insulation system lost $50-60 \%$ of their room temperature prestress during cool down as measured with strain gauges monitoring the azimuthal prestress in the magnets. This effect was improved by including a mineral filler in one layer of the cable wrap. Magnets built with the CI system, in which the outer layer of coil insulation is a filled material, lose typically 40-50\% of their room temperature prestress, a loss very similar to the loss experienced by K/FG magnets upon cool down and not detrimental to ultimate magnet performance.
Kapton CI coils were initially molded by bringing their temperature up to $225 \mathrm{C}$, then back to room temperature, while maintaining a pressure on the coils of nominally 10 $\mathrm{kpsi}$ in a molding press. It was found that coils made in this way exhibited low interstrand resistance and magnets built with these coils experienced strong and undesirable ramprate-dependent effects ${ }^{[3]}$. A modified molding program in which the pressure on the coils was removed during the two temperature ramps between $135 \mathrm{C}$ and $225 \mathrm{C}$ and reduced to a low value at the $225 \mathrm{C}$ temperature plateau was found to ameliorate this effect. In this modified program, coil sizing is accomplished by maintaining high pressure on the coil for $30 \mathrm{~min}$. while at a temperature of $135 \mathrm{C}$ on both the up and down temperature cycles. The interstrand resistance of coils made in this way is similar to that of $\mathrm{K} / \mathrm{FG}$ coils made with the traditional cycle of $7 \mathrm{kpsi}$ pressure during a temperature cycle to $135 \mathrm{C}$, and in some cases has been found to result in a higher level of interstrand resistance ${ }^{[4]}$. Extensive investigations into the general question of interstrand resistance have revealed it to be a poorly understood phenomenon dependent on numerous factors including cable manufacturer, manufacturing process, level of oxidation of the wire in the cable, etc., and it has become the subject of extensive investigations in the SSC and RHIC magnet programs. It has been found that the interstrand resistance is quite reproducible if the manufacturing process for the cable is held constant. Thus, magnets that show repeatable and acceptable eddy current effects can be manufactured with either $\mathrm{K} / \mathrm{FG}$ or $\mathrm{CI}$ cable insulation if the cable characteristics are controlled.

\section{SUMMARY}

The Kapton CI cable insulation system described in this paper has been shown through extensive testing to be robust and reliable. It does require higher molding temperatures but these can readily be accommodated through proper design of the coil tooling. Its characteristics are equal to or superior in all known respects to those of the Kapton/Fiberglass system that has been used to date. In particular, its improved punch-through resistance gives a significant reduction in the probability of electrical faults in the current generation of high field, high prestress accelerator magnets, a characteristic that will be critical for successful operation of large machines built of such magnets.

\section{REFERENCES}

[1] A. Devred et al, "About the Mechanics of SSC Dipole Magnet Prototypes", AIP Conference Proceedings: The Physics of Particle Accelerators, January 1992 and SSCL-Preprint-6, November, 1991.

[2] W. Sampson, private communication.

[3] P. Wanderer, "Test Results from DSA209", presentation at SSC Magnet Systems Integration Meeting, May, 1991 (unpublished).

[4] M.D. Anerella, A.K. Ghosh, SSC Internal Tech. Note SSC-MD-273, (1991). 